\title{
Research Article \\ Dynamics of a Discrete Internet Congestion Control Model
}

\section{Yingguo Li}

School of Mathematics and Computer Science, Fujian Normal University, Fuzhou 350007, China

Correspondence should be addressed to Yingguo Li, yguoli@fjnu.edu.cn

Received 24 July 2011; Accepted 1 September 2011

Academic Editor: Carlo Piccardi

Copyright (C) 2011 Yingguo Li. This is an open access article distributed under the Creative Commons Attribution License, which permits unrestricted use, distribution, and reproduction in any medium, provided the original work is properly cited.

We consider a discrete Internet model with a single link accessed by a single source, which responds to congestion signals from the network. Firstly, the stability of the equilibria of the system is investigated by analyzing the characteristic equation. By choosing the time delay as a bifurcation parameter, we prove that Neimark-Sacker bifurcations occur when the delay passes a sequence of critical values. Then, the explicit algorithm for determining the direction of the Neimark-Sacker bifurcations and the stability of the bifurcating periodic solutions is derived. Finally, some numerical simulations are given to verify the theoretical analysis.

\section{Introduction}

Congestion control algorithms and active queue management (AQM) for Internet have been the focus of intense research since the seminal work of Kelly et al. [1]. The primal algorithms they have introduced are based on explicit feedback functions, where the sum of user utilities is maximized within the capacity constraints of the links. In primal algorithms, the users adapt the source rates dynamically based on the congestion signal generated by the link, and the links select a static law to determine the link prices directly from the arrival rates at the links [2]. In [3], a stability condition was provided for a single proportionally fair congestion controller with delayed feedback. Since then, this result was extended to networks in $[4,5]$ and for different classes of controllers in [6].

In this paper, we consider an Internet model with a single link and single source, which can be formulated as a congestion control system with feedback delay. The model is described by $[3,7,8]$

$$
x^{\prime}(t)=k[w-x(t-\tau) p(x(t-\tau))]
$$

where $x(t)$ is the sending rate of the source at time $t, k$ is a positive gain parameter, $\tau$ is the sum of forward and return delays, $w>0$ is a target (set point), and the congestion indication 
function $p(\cdot)$ is increasing, nonnegative, and not identically zero, which can be viewed as the probability that a packet at the resource receives a feedback congestion indication signal. System (1.1) corresponds to a rate control algorithm for the user, which can be used to adjust the sending rate $x(t)$ so that the expected number of signals received by the user will tend to target $w$. A great deal of research has been devoted to the asymptotic behaviour, periodic solutions, global stability, and other properties of this model, for which, we refer to [3, 7-9].

But considering the need of scientific computation and real-time simulation, our interest is focused on the behaviors of discrete dynamics system corresponding to (1.1). Many authors considered the numerical approximation of a scalar delay differential equation by using different numerical methods, such as $\theta$-method, Euler method, and Trapezoidal method (see [10-15]). In this paper, we use the forward Euler scheme to make the discretization for system (1.1).

Moreover, it is also of interest to find what will happen when the congestion control system loses stability. The purpose of this paper is to discuss this version as a discrete dynamical system by using Neimark-Sacker bifurcation theory of discrete systems. We not only investigate the stability of the fixed point and the existence of the Neimark-Sacker bifurcations, but also the stability and direction of the Neimark-Sacker bifurcation of the discrete system.

The paper is organized as follows: in Section 2, we analyze the distribution of the characteristic equation associated with the discrete model and obtain the existence of the local Neimark-Sacker bifurcation. In Section 3, the direction and stability of closed invariant curve from the Neimark-Sacker bifurcation of the discrete delay model are determined by using the theories of discrete systems in [16]. In the final section, some computer simulations are performed to illustrate the analytical results found.

\section{Stability Analysis}

Let $u(t)=x(t \tau)$. Then (1.1) can be rewritten as

$$
u^{\prime}(t)=\tau k[w-u(t-1) p(u(t-1))]
$$

We consider sufficient small step size of the form $h=1 / m$, where $m \in \mathbb{Z}_{+}$. The Euler method applied to this equation yields the delay difference equation

$$
u_{n+1}=u_{n}+\tau k h\left[w-u_{n-m} p\left(u_{n-m}\right)\right]
$$

where $u_{n}$ is an approximate value to $u(n h)$. Let $u^{*}$ be a positive equilibrium of $(2.2)$, then we have

$$
u^{*} p\left(u^{*}\right)=w
$$

Set $y_{n}=u_{n}-u^{*}$, then there follows:

$$
y_{n+1}=y_{n}+\tau k h\left[w-\left(y_{n-m}+u^{*}\right) p\left(y_{n-m}+u^{*}\right)\right] .
$$


By introducing a new variable $Y_{n}=\left(y_{n}, y_{n-1}, \ldots, y_{n-m}\right)^{T}$, we can rewrite (2.4) in the form

$$
Y_{n+1}=F\left(Y_{n}, \tau\right)
$$

where $F=\left(F_{0}, F_{1}, \ldots, F_{m}\right)^{T}$, and

$$
F_{j}= \begin{cases}y_{n}+\tau k h\left[w-\left(y_{n-m}+u^{*}\right) p\left(y_{n-m}+u^{*}\right)\right], & j=0 \\ y_{n-j+1}, & 1 \leq j \leq m .\end{cases}
$$

Clearly the origin is a fixed point of (2.5), and the linear part of (2.5) is

$$
Y_{n+1}=A Y_{n}
$$

where

$$
A=\left(\begin{array}{ccccc}
1 & 0 & \cdots & 0 & -\tau k h\left[p\left(u^{*}\right)+u^{*} p^{\prime}\left(u^{*}\right)\right] \\
1 & 0 & \cdots & 0 & 0 \\
0 & 1 & \cdots & 0 & 0 \\
\vdots & \vdots & \ddots & \vdots & \vdots \\
0 & 0 & \vdots & 1 & 0
\end{array}\right)
$$

The characteristic equation of $A$ is given by

$$
a(\lambda):=\lambda^{m+1}-\lambda^{m}+\tau k h\left[p\left(u^{*}\right)+u^{*} p^{\prime}\left(u^{*}\right)\right]=0 .
$$

It is well known that the stability of the zero equilibrium solution of (2.5) depends on the distribution of the zeros of the roots of (2.9). In order to proof the existence of the local Neimark-Sacker bifurcation at fixed point, we need some lemmas as follows.

Lemma 2.1. There exists a $\bar{\tau}>0$ such that for $0<\tau<\bar{\tau}$ all roots of (2.9) have modulus less than one.

Proof. When $\tau=0$, (2.9) becomes

$$
\lambda^{m+1}-\lambda^{m}=0
$$

The equation has, at $\tau=0$, an $m$-fold root $\lambda=0$ and a simple root $\lambda=1$. 
Consider the root $\lambda(\tau)$ such that $\lambda(0)=1$. This root depends continuously on $\tau$ and is a differential function of $\tau$. From (2.9), we have

$$
\begin{aligned}
& \frac{d \lambda}{d \tau}=\frac{k h\left[p\left(u^{*}\right)+u^{*} p^{\prime}\left(u^{*}\right)\right]}{m \lambda^{m-1}-(m+1) \lambda^{m}}, \\
& \frac{d \bar{\lambda}}{d \tau}=\frac{k h\left[p\left(u^{*}\right)+u^{*} p^{\prime}\left(u^{*}\right)\right]}{m \bar{\lambda}^{m-1}-(m+1) \bar{\lambda}^{m}} .
\end{aligned}
$$

Since $p(\cdot)$ is increasing and nonnegative, we have

$$
\left.\frac{d|\lambda|^{2}}{d \tau}\right|_{\tau=0, \lambda=1}=\left.\left[\lambda \frac{d \bar{\lambda}}{d \tau}+\bar{\lambda} \frac{d \lambda}{d \tau}\right]\right|_{\tau=0, \lambda=1}=-2 k h\left[p\left(u^{*}\right)+u^{*} p^{\prime}\left(u^{*}\right)\right]<0
$$

So with the increasing of $\tau>0, \lambda$ cannot cross $\lambda=1$. Consequently, all roots of (2.9) lie in the unit circle for sufficiently small positive $\tau>0$, and the existence of the $\bar{\tau}$ follows.

A Neimark-Sacker bifurcation occurs when a complex conjugate pair of eigenvalues of $A$ cross the unit circle as $\tau$ varies. We have to find values of $\tau$ such that there are roots on the unit circle. Denote the roots on the unit circle by $e^{i \omega}, \omega \in(-\pi, \pi]$. Since we are dealing with complex roots of a real polynomial, we only need to look for $\omega \in(0, \pi]$. For $\omega \in(0, \pi]$, $e^{i \omega}$ is a root of (2.9) if and only if

$$
e^{i \omega}-1+\tau k h\left[p\left(u^{*}\right)+u^{*} p^{\prime}\left(u^{*}\right)\right] e^{-i m \omega}=0
$$

Separating the real part and imaginary part from (2.13), there are

$$
\begin{aligned}
& \cos \omega+\tau k h\left[p\left(u^{*}\right)+u^{*} p^{\prime}\left(u^{*}\right)\right] \cos m \omega=1, \\
& \sin \omega-\tau k h\left[p\left(u^{*}\right)+u^{*} p^{\prime}\left(u^{*}\right)\right] \sin m \omega=0 .
\end{aligned}
$$

So

$$
\cos \omega=1-\frac{1}{2}(\tau k h)^{2}\left[p\left(u^{*}\right)+u^{*} p^{\prime}\left(u^{*}\right)\right]^{2}
$$

From (2.14) and (2.15), we obtain that

$$
\sin m \omega \cos \omega+\cos m \omega \sin \omega=\sin m \omega,
$$

that is,

$$
\sin (m+1) \omega=\sin m \omega
$$


then there exists a sequence of

$$
\omega_{j} \in\left(\frac{2 j \pi}{m}, \frac{(2 j+1) \pi}{m}\right), \quad j=0,1,2, \ldots,\left[\frac{m-1}{2}\right]
$$

where [-] denotes the greatest integer function. It is clear that there exists a sequence of the time delay parameters $\tau_{j}$ satisfying (2.14) and (2.15), according to $\omega=\omega_{j}$.

From (2.15), we have

$$
\tau_{j}=\tau\left(\omega_{j}\right)=\frac{\sin \omega_{j}}{k h\left[p\left(u^{*}\right)+u^{*} p^{\prime}\left(u^{*}\right)\right] \sin m \omega_{j}} .
$$

Differentiating (2.16) with respect to $\omega$, we obtain that

$$
\sin \omega=\tau\left\{k h\left[p\left(u^{*}\right)+u^{*} p^{\prime}\left(u^{*}\right)\right]\right\}^{2} \frac{d \tau}{d \omega} .
$$

This gives

$$
\left.\frac{d \tau}{d \omega}\right|_{\omega=\omega_{j}, \tau=\tau_{j}}=\frac{\sin \omega_{j}}{\tau_{j}\left\{k h\left[p\left(u^{*}\right)+u^{*} p^{\prime}\left(u^{*}\right)\right]\right\}^{2}}>0 .
$$

Therefore,

$$
0<\tau_{0}<\tau_{1}<\cdots<\tau_{[(m-1) / 2]}
$$

Lemma 2.2. Let $\lambda_{j}(\tau)=r_{j}(\tau) e^{i \omega_{j}(\tau)}$ be a root of $(2.9)$ near $\tau=\tau_{j}$ satisfying $r_{j}\left(\tau_{j}\right)=1$ and $\omega_{j}\left(\tau_{j}\right)=$ $\omega_{j}$. Then

$$
\left.\frac{d r_{j}^{2}(\tau)}{d \tau}\right|_{\tau=\tau_{j}, \omega=\omega_{j}}>0
$$

Proof. From (2.11), we have

$$
\begin{aligned}
\left.\frac{d r_{j}^{2}}{d \tau}\right|_{\tau=\tau_{j}, \omega=\omega_{j}} & =\left.\frac{d\left|\lambda_{j}\right|^{2}}{d \tau}\right|_{\tau=\tau_{j}, \omega=\omega_{j}}=\left.\left[\lambda \frac{d \bar{\lambda}}{d \tau}+\bar{\lambda} \frac{d \lambda}{d \tau}\right]\right|_{\tau=\tau_{j}, \omega=\omega_{j}} \\
& =\frac{2 k h\left[p\left(u^{*}\right)+u^{*} p^{\prime}\left(u^{*}\right)\right]\left[m \cos m \omega_{j}-(m+1) \cos (m+1) \omega_{j}\right]}{\left|m e^{i(m-1) \omega_{j}}-(m+1) e^{i m \omega_{j}}\right|^{2}} .
\end{aligned}
$$


From (2.14) and (2.15), we obtain that

$$
\begin{aligned}
\cos m \omega_{j} & =\frac{1-\cos \omega_{j}}{\tau_{j} k h\left[p\left(u^{*}\right)+u^{*} p^{\prime}\left(u^{*}\right)\right]}, \\
\sin m \omega_{j} & =\frac{\sin \omega_{j}}{\tau_{j} k h\left[p\left(u^{*}\right)+u^{*} p^{\prime}\left(u^{*}\right)\right]} .
\end{aligned}
$$

It is easy to see that

$$
\begin{aligned}
\cos (m+1) \omega_{j} & =\cos m \omega_{j} \cos \omega_{j}-\sin m \omega_{j} \sin \omega_{j} \\
& =\frac{\cos \omega_{j}-1}{\tau k h\left[p\left(u^{*}\right)+u^{*} p^{\prime}\left(u^{*}\right)\right]} .
\end{aligned}
$$

Using (2.25)-(2.27), we have

$$
\left.\frac{d\left|\lambda_{j}\right|^{2}}{d \tau}\right|_{\tau=\tau_{j}, \omega=\omega_{j}}=\frac{2(2 m+1)\left(1-\cos \omega_{j}\right)}{\tau_{j}\left|m e^{i(m-1) \omega_{j}}-(m+1) e^{i m \omega_{j}}\right|^{2}}>0 .
$$

This completes the proof.

Lemma 2.3. Equation (2.9) has a pair of simple roots $e^{ \pm i \omega_{j}}$ on the unit circle when $\tau=\tau_{j}, j=$ $0,1,2, \ldots,[(m-1) / 2]$. Furthermore, if $\tau \in\left[0, \tau_{0}\right)$, then all the roots of $(2.9)$ have modulus less than one; If $\tau=\tau_{0}$, then all roots of (2.9) except $e^{ \pm i \omega_{0}}$ have modulus less than one. But if $\tau \in\left(\tau_{j}, \tau_{j+1}\right]$, for $j=0,1,2, \ldots,[(m-1) / 2],(2.9)$ has $2(j+1)$, roots have modulus more than one.

Proof. From (2.18)-(2.20), we have that (2.9) has toots $e^{ \pm i \omega_{j}}$ if and only if $\tau=\tau_{j}$ and $\omega=\omega_{j}$ given in (2.19) and (2.20). By Lemmas 2.1 and 2.2, we know that if $\tau \in\left[0, \tau_{0}\right)$, then all roots of (2.9) have modulus less than one; if $\tau=\tau_{0}$, then all roots of (2.9) except $e^{ \pm i \omega_{j}}$ have modulus less than one; furthermore, by the Rouchs theorem (Dieudonné [17, Theorem 9.17.4]), the statement on the number of eigenvalues with modulus more than one is as follows.

Lemma 2.3 immediately leads to stability of the zero solution of (2.4), and equivalently, of the positive fixed point $u=u^{*}$ of (2.2). So we have the following results on stability and bifurcation in system (2.2).

Theorem 2.4. There exists a sequence of values of the time delay parameter $0<\tau_{0}<\tau_{1}<\cdots<$ $\tau_{[(m-1) / 2]}$ such that the positive fixed point $u=u^{*}$ of (2.2) is asymptotically stable for $\tau \in\left[0, \tau_{0}\right)$ and unstable for $\tau>\tau_{0}$. Equation (2.2) undergoes a Neimark-Sacker bifurcation at the $u^{*}$ when $\tau=\tau_{j}, j=0,1,2, \ldots,[(m-1) / 2]$, where $\tau_{j}$ satisfies (2.20).

\section{Direction and Stability of the Neimark-Sacker Bifurcation in Discrete Model}

In the previous section, we obtain the conditions under which a family of periodic solutions bifurcate from the steady state at the critical value $\tau=\tau_{j}, j=0,1,2, \ldots,[(m-1) / 2]$. Without loss of generality, denote the critical value $\tau=\tau_{j}$ by $\tau^{*}$. For continuous systems, as pointed 
out in Hassard et al. [18], it is interesting and important to determine the direction, stability, and the period of these periodic solutions bifurcating from the steady state. In this section, following the idea of Hassard et al., we shall study the direction, stability, and the period of the bifurcating periodic solution when $\tau=\tau_{0}$ in the discrete Internet congestion control model. The method we used is based on the theories of discrete system by Kuznetsov [16].

Set $\tau=\tau_{0}+v, v \in \mathbb{R}$. Then $v=0$ is a Neimark-Sacker bifurcation value for (2.4). Rewrite (2.4) as

$$
\begin{gathered}
y_{n+1}=y_{n}-\tau k h\left[\left(p\left(u^{*}\right)+u^{*} p^{\prime}\left(u^{*}\right)\right) y_{n-m}+\frac{1}{2}\left(2 p^{\prime}\left(u^{*}\right)+u^{*} p^{\prime \prime}\left(u^{*}\right)\right) y_{n-m}^{2}\right. \\
\left.+\frac{1}{6}\left(3 p^{\prime \prime}\left(u^{*}\right)+u^{*} p^{\prime \prime \prime}\left(u^{*}\right)\right) y_{n-m}^{3}\right]+O\left(\left|y_{n-m}\right|^{4}\right) .
\end{gathered}
$$

So system (2.5) is turned into

$$
Y_{n+1}=A Y_{n}+\frac{1}{2} B\left(Y_{n}, Y_{n}\right)+\frac{1}{6} C\left(Y_{n}, Y_{n}, Y_{n}\right)+O\left(\left\|Y_{n}\right\|^{4}\right)
$$

where

$$
\begin{gathered}
B\left(Y_{n}, Y_{n}\right)=\left(b_{0}\left(Y_{n}, Y_{n}\right), 0, \ldots, 0\right), \quad C\left(Y_{n}, Y_{n}, Y_{n}\right)=\left(c_{0}\left(Y_{n}, Y_{n}, Y_{n}\right), 0, \ldots, 0\right), \\
b_{0}(\phi, \psi)=-\tau k h\left(2 p^{\prime}\left(u^{*}\right)+u^{*} p^{\prime \prime}\left(u^{*}\right)\right) \phi_{m} \psi_{m}, \\
c_{0}\left(\phi_{1}, \psi, \eta\right)=-\tau k h\left(3 p^{\prime \prime}\left(u^{*}\right)+u^{*} p^{\prime \prime \prime}\left(u^{*}\right)\right) \phi_{m} \psi_{m} \eta_{m} .
\end{gathered}
$$

Let $q=q\left(\tau_{0}\right) \in \mathbb{C}^{m+1}$ be an eigenvector of $A$ corresponding to $e^{i \omega_{0}}$, then

$$
A q=e^{i \omega_{0}} q, \quad A \bar{q}=e^{-i \omega_{0}} \bar{q}
$$

We also introduce an adjoint eigenvector $q^{*}=q^{*}(\tau) \in \mathbb{C}^{m+1}$ having the properties

$$
A^{T} q^{*}=e^{-i \omega_{0}} q^{*}, \quad A^{T} \bar{q}^{*}=e^{i \omega_{0}} \bar{q}^{*}
$$

and satisfying the normalization $\left\langle q^{*}, q\right\rangle=1$, where $\left\langle q^{*}, q\right\rangle=\sum_{i=0}^{m} \bar{q}_{i}^{*} q_{i}$.

Lemma 3.1 (see [19]). Define a vector-valued function $q: \mathbb{C} \rightarrow \mathbb{C}^{m+1}$ by

$$
p(\xi)=\left(\xi^{m}, \xi^{m-1}, \ldots, 1\right)^{T}
$$

If $\xi$ is an eigenvalue of $A$, then $A p(\xi)=\xi p(\xi)$.

In view of Lemma 3.1, we have

$$
q=p\left(e^{i \omega_{0}}\right)=\left(e^{i m \omega_{0}}, e^{i(m-1) \omega_{0}}, \ldots, e^{i \omega_{0}}, 1\right)^{T}
$$


Lemma 3.2. Suppose that $q^{*}=\left(q_{0}^{*}, q_{1}^{*}, \ldots, q_{m}^{*}\right)^{T}$ is the eigenvector of $A^{T}$ corresponding to eigenvalue $e^{-i \omega_{0}}$, and $\left\langle q^{*}, q\right\rangle=1$. Then

$$
q^{*}=\bar{K}\left(1, a_{0} e^{i m \omega_{0}}, a_{0} e^{i(m-1) \omega_{0}}, \ldots, a_{0} e^{i 2 \omega_{0}}, a_{0} e^{i \omega_{0}}\right)^{T}
$$

where $a_{0}=-\tau k h\left(2 p^{\prime}\left(u^{*}\right)+u^{*} p^{\prime \prime}\left(u^{*}\right)\right)$ and

$$
K=\left(e^{i m \omega_{0}}+m a_{0} e^{-i \omega_{0}}\right)^{-1}
$$

Proof. Assign $q^{*}$ satisfies $A^{T} q^{*}=\bar{z} q^{*}$ with $\bar{z}=e^{-i \omega_{0}}$, then the following identities hold

$$
\begin{gathered}
q_{0}^{*}+q_{1}^{*}=e^{-i \omega_{0}} q_{0}^{*}, \\
q_{k}^{*}=e^{-i \omega_{0}} q_{k-1}^{*}, \quad k=2, \ldots, m, \\
a_{0} q_{0}^{*}=e^{-i \omega_{0}} q_{m}^{*} .
\end{gathered}
$$

Let $q_{m}^{*}=a_{0} e^{i \omega_{0}} \bar{K}$, then

$$
q^{*}=\bar{K}\left(1, a_{0} e^{i m \omega_{0}}, a_{0} e^{i(m-1) \omega_{0}}, \ldots, a_{0} e^{i 2 \omega_{0}}, a_{0} e^{i \omega_{0}}\right)^{T} .
$$

From normalization $\left\langle q^{*}, q\right\rangle=1$ and computation, (3.10) is held.

Let $a(\lambda)$ be characteristic polynomial of $A$ and $\lambda=e^{i \omega_{0}}$. Following the algorithms in [16] and using a computation process similar to that in [19], we can compute an expression for the critical coefficient $c_{1}\left(\tau_{0}\right)$,

$$
c_{1}\left(\tau_{0}\right)=\frac{g_{20} g_{11}\left(1-2 \lambda_{0}\right)}{2\left(\lambda_{0}^{2}-\lambda_{0}\right)}+\frac{\left|g_{11}\right|^{2}}{1-\bar{\lambda}_{0}}+\frac{\left|g_{02}\right|^{2}}{2\left(\lambda_{0}^{2}-\bar{\lambda}_{0}\right)}+\frac{g_{21}}{2},
$$

where

$$
\begin{gathered}
g_{20}=\left\langle q^{*}, B(q, q)\right\rangle, \quad g_{11}=\left\langle q^{*}, B(q, \bar{q})\right\rangle, \quad g_{02}=\left\langle q^{*}, B(\bar{q}, \bar{q})\right\rangle, \\
g_{21}=\left\langle q^{*}, B\left(\bar{q}, \omega_{20}\right)\right\rangle+2\left\langle q^{*}, B\left(q, \omega_{11}\right)\right\rangle+\left\langle q^{*}, C(q, q, \bar{q})\right\rangle, \\
\omega_{20}=\frac{b_{0}(q, q)}{a\left(\lambda_{0}^{2}\right)} p\left(\lambda_{0}^{2}\right)-\frac{\left\langle q^{*}, B(q, q)\right\rangle}{\lambda_{0}^{2}-\lambda_{0}} q-\frac{\left\langle\bar{q}^{*}, B(q, q)\right\rangle}{\lambda_{0}^{2}-\bar{\lambda}_{0}} \bar{q}, \\
\omega_{11}=\frac{b_{0}(q, \bar{q})}{a(1)} p(1)-\frac{\left\langle q^{*}, B(q, \bar{q})\right\rangle}{1-\lambda_{0}} q-\frac{\left\langle\bar{q}^{*}, B(q, \bar{q})\right\rangle}{1-\bar{\lambda}_{0}} \bar{q} .
\end{gathered}
$$


By (3.4), (3.8), and Lemma 3.2, we get

$$
\begin{gathered}
b_{0}\left(\bar{q}, p\left(e^{i 2 \omega_{0}}\right)\right)=-\tau k h\left(2 p^{\prime}\left(u^{*}\right)+u^{*} p^{\prime \prime}\left(u^{*}\right)\right), \\
b_{0}(q, q)=-\tau k h\left(2 p^{\prime}\left(u^{*}\right)+u^{*} p^{\prime \prime}\left(u^{*}\right)\right), \\
b_{0}(q, \bar{q})=-\tau k h\left(2 p^{\prime}\left(u^{*}\right)+u^{*} p^{\prime \prime}\left(u^{*}\right)\right), \\
c_{0}(q, q, \bar{q})=-\tau k h\left(3 p^{\prime \prime}\left(u^{*}\right)+u^{*} p^{\prime \prime \prime}\left(u^{*}\right)\right), \\
a\left(e^{i 2 \omega_{0}}\right)=e^{i 2(m+1) \omega_{0}}-e^{i 2 m \omega_{0}}+\tau k h\left(2 p^{\prime}\left(u^{*}\right)+u^{*} p^{\prime \prime}\left(u^{*}\right)\right), \\
a(1)=\tau k h\left(2 p^{\prime}\left(u^{*}\right)+u^{*} p^{\prime \prime}\left(u^{*}\right)\right), \\
b_{0}(q, p(1))=-\tau k h\left(2 p^{\prime}\left(u^{*}\right)+u^{*} p^{\prime \prime}\left(u^{*}\right)\right) .
\end{gathered}
$$

Substituting these into (3.13), we have

$$
\begin{aligned}
c_{1}\left(\tau_{0}\right)=\frac{K}{2}\left\{\frac{\left[\tau k h\left(2 p^{\prime}\left(u^{*}\right)+u^{*} p^{\prime \prime}\left(u^{*}\right)\right)\right]^{2}}{a\left(e^{i 2 \omega_{0}}\right)}+\frac{2\left[\tau k h\left(2 p^{\prime}\left(u^{*}\right)+u^{*} p^{\prime \prime}\left(u^{*}\right)\right)\right]^{2}}{a(1)}\right. \\
\left.-\tau k h\left(3 p^{\prime \prime}\left(u^{*}\right)+u^{*} p^{\prime \prime \prime}\left(u^{*}\right)\right)\right\} .
\end{aligned}
$$

Lemma 3.3 (see [20]). Given the map (2.5) and assume that

(1) $\lambda(\tau)=r(\tau) e^{i \omega(\tau)}$, where $r\left(\tau^{*}\right)=1, r^{\prime}\left(\tau^{*}\right) \neq 0$ and $\omega\left(\tau^{*}\right)=\omega^{*}$;

(2) $e^{i k \omega^{*}} \neq 1$ for $k=1,2,3,4$;

(3) $\operatorname{Re}\left[e^{-i \omega^{*}} c_{1}\left(\tau^{*}\right)\right] \neq 0$.

Then an invariant closed curve, topologically equivalent to a circle, for map (2.5) exists for $\tau$ in a one-side neighborhood of $\tau^{*}$. The radius of the invariant curve grows like $O\left(\sqrt{\left|\tau-\tau^{*}\right|}\right)$. One of the four cases below applies:

(1) $r^{\prime}\left(\tau^{*}\right)>0, \operatorname{Re}\left[e^{-i \omega^{*}} c_{1}\left(\tau^{*}\right)\right]<0$. The origin is asymptotically stable for $\tau<\tau^{*}$ and unstable for $\tau>\tau^{*}$. An attracting invariant closed curve exists for $\tau>\tau^{*}$.

(2) $r^{\prime}\left(\tau^{*}\right)>0, \operatorname{Re}\left[e^{-i \omega^{*}} c_{1}\left(\tau^{*}\right)\right]>0$. The origin is asymptotically stable for $\tau<\tau^{*}$ and unstable for $\tau>\tau^{*}$. An repelling invariant closed curve exists for $\tau<\tau^{*}$.

(3) $r^{\prime}\left(\tau^{*}\right)<0, \operatorname{Re}\left[e^{-i \omega^{*}} c_{1}\left(\tau^{*}\right)\right]<0$. The origin is asymptotically stable for $\tau>\tau^{*}$ and unstable for $\tau<\tau^{*}$. An attracting invariant closed curve exists for $\tau<\tau^{*}$.

(4) $r^{\prime}\left(\tau^{*}\right)<0, \operatorname{Re}\left[e^{-i \omega^{*}} c_{1}\left(\tau^{*}\right)\right]>0$. The origin is asymptotically stable for $\tau>\tau^{*}$ and unstable for $\tau<\tau^{*}$. An repelling invariant closed curve exists for $\tau>\tau^{*}$.

From the discussion in Section 2, we know that $r^{\prime}\left(\tau^{*}\right)>0$; therefore, by Lemma 3.3, we have the following result. 


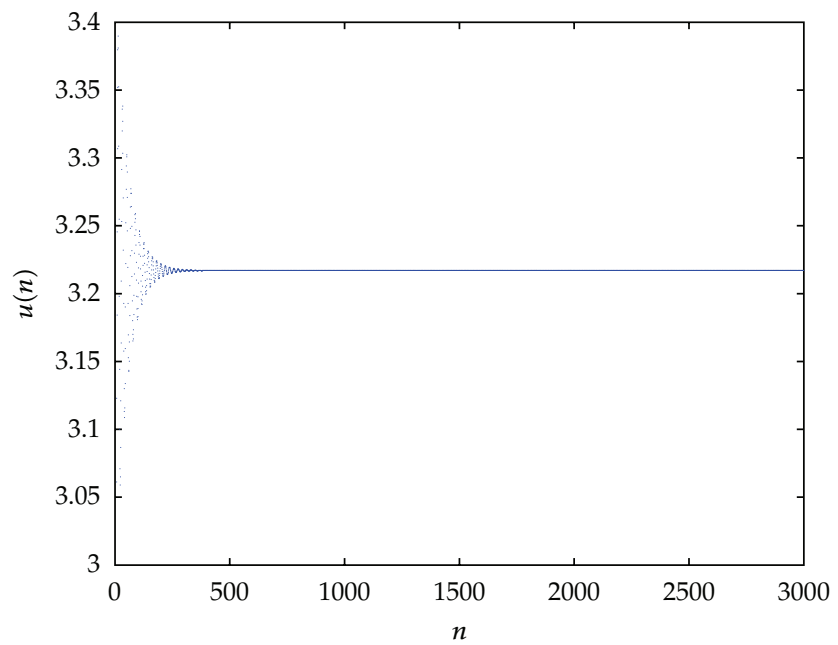

(a)

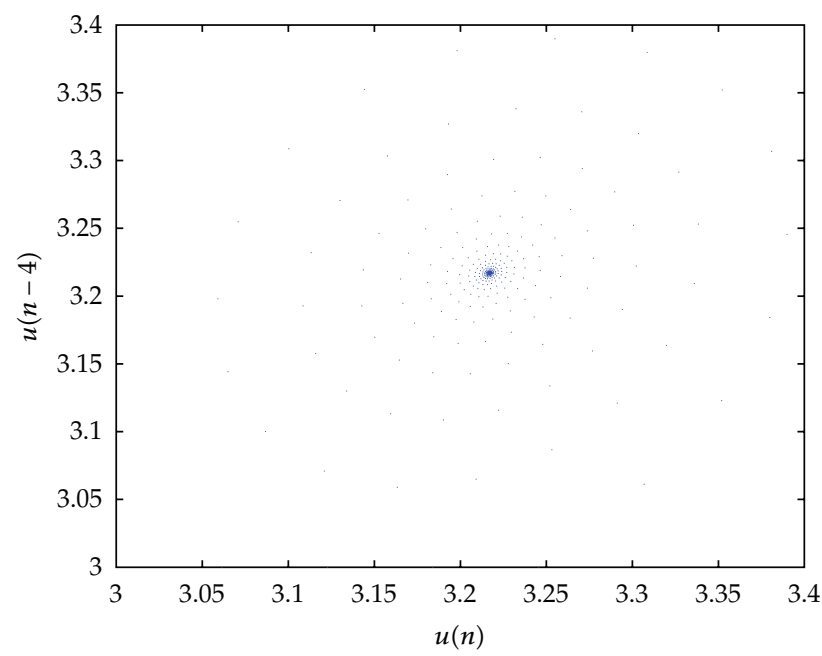

(b)

Figure 1: (a) The equilibrium $u^{*}$ of (2.2) is asymptotically stable for $\tau=0.9<\tau_{0}=1.0159$, (b) Phase plot of (2.2) on the plane $(u(n), u(n-4))$ for $\tau=0.9<\tau_{0}=1.0159$.

Theorem 3.4. For (2.2), $u=u^{*}$ is asymptotically stable for $\tau \in\left[0, \tau_{0}\right)$ and unstable for $\tau>\tau_{0}$. An attracting (repelling) invariant closed curve exists for $\tau>\tau_{0}$ if $\operatorname{Re}\left[e^{-i \omega_{0}} c_{1}\left(\tau_{0}\right)\right]<0(>0)$.

\section{Computer Simulation}

In this section, we will confirm our theoretical analysis by numerical simulation. We choose $p(x)=x /(20-3 x)$ as in [8] and set $k=1.5, m=4, w=1$. Then $\tau_{0}=1.0159$ is the NeimarkSacker bifurcation value.

Figures 1 and 2 are about delay difference equation (2.2) when step size $h=0.25$.

In Figure 1, we show the waveform plot and phase plot for (2.2) with initial values $u_{j}=3(j=0,1, \ldots, 4)$ for $\tau=0.9<\tau_{0}=1.0159$. The equilibrium $u^{*}=3.2170$ of $(2.2)$ 


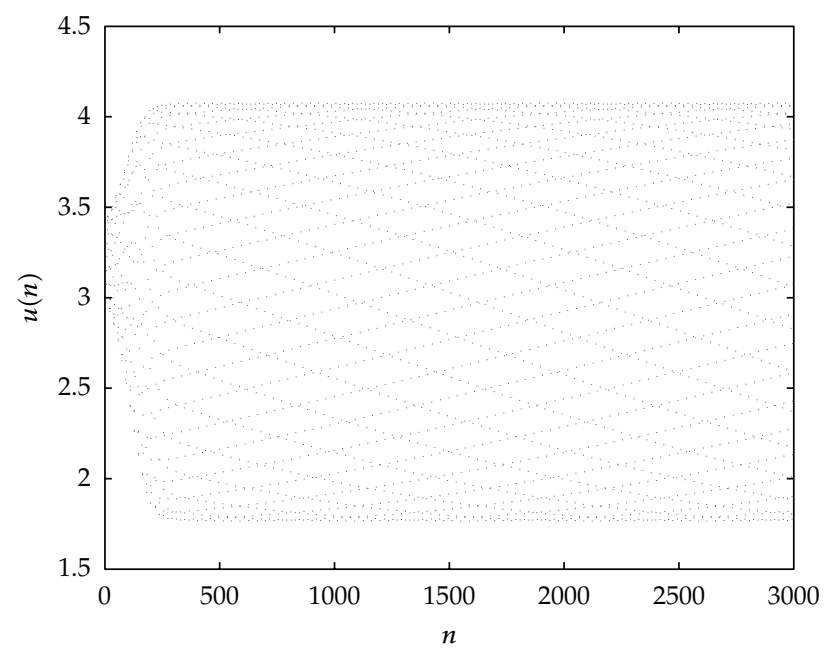

(a)

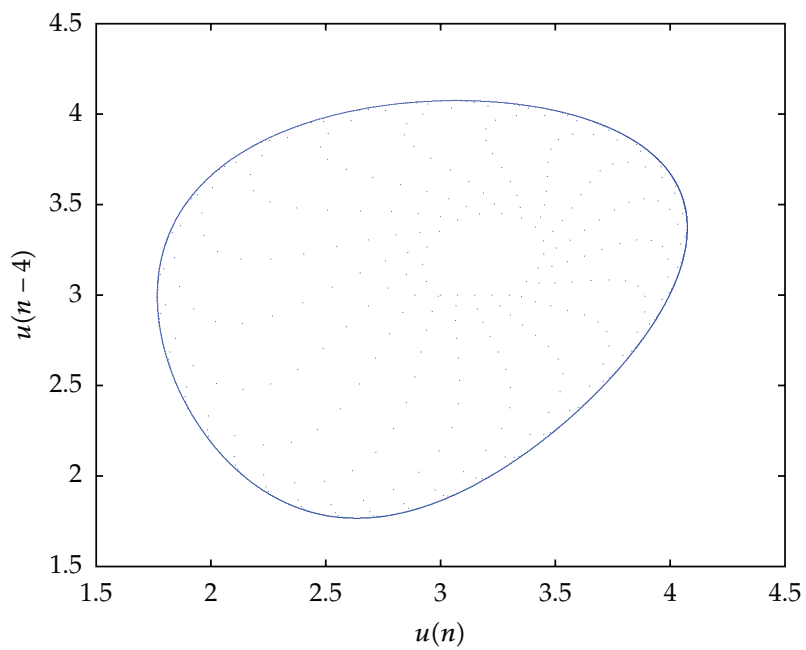

(b)

Figure 2: (a) Waveform plot of (2.2) for $\tau=1.1>\tau_{0}=1.0159$, (b) A bifurcating periodic solution appears on the plane $(u(n), u(n-4))$ for $\tau=1.1>\tau_{0}=1.0159$.

is asymptotically stable. In Figure 2, we show the waveform plot and phase plot for (2.2) with initial values $u_{j}=3(j=0,1, \ldots, 4)$. The equilibrium $u^{*}=3.2170$ of (2.2) is unstable for $\tau=1.1>\tau_{0}=1.0159$. When $\tau$ varies and passes through $\tau_{0}=1.0159$, the equilibrium loses its stability and a periodic solution bifurcates from the equilibrium for $\tau=1.1>\tau_{0}=$ 1.0159. That is the delay difference equation (2.2) which has a Neimark-Sacker bifurcation at $\tau_{0}$.

\section{Acknowledgment}

The authors are very grateful to the referees for their valuable suggestions, which help to improve the paper significantly. 


\section{References}

[1] F. P. Kelly, A. K. Maulloo, and D. Tan, "Rate control for communication networks: Shadow prices, proportional fairness and stability," Journal of the Operational Research Society, vol. 49, no. 3, pp. 237252, 1998.

[2] T. Alpcan and T. Basar, "A globally stable adaptive congestion control scheme for internet-style networks with delay," IEEE/ACM Transactions on Networking, vol. 13, no. 6, pp. 1261-1274, 2005.

[3] F. P. Kelly, "Models for a self-managed Internet," Philosophical Transactions of the Royal Society A, vol. 358, no. 1773, pp. 2335-2348, 2000.

[4] L. Massoulié, "Stability of distributed congestion control with heterogeneous feedback delays," IEEE. Transactions on Automatic Control, vol. 47, no. 6, pp. 895-902, 2002.

[5] X. F. Wang, G. Chen, and K.-T. Ko, "A stability theorem for internet congestion control," Systems $\mathcal{E}$ Control Letters, vol. 45, no. 2, pp. 81-85, 2002.

[6] F. Paganini, J. Doyle, and S. Low, "Scalable laws for stable network congestion control," in Proceedings of the IEEE Conference on Decision and Control, vol. 1, pp. 185-190, 2001.

[7] S. Deb and R. Srikant, "Global stability of congestion controllers for the internet," in Proceedings of the IEEE Conference on Decision and Control, vol. 4, pp. 3626-3631, 2002.

[8] C. Li, G. Chen, X. Liao, and J. Yu, "Hopf bifurcation in an internet congestion control model," Chaos, Solitons and Fractals, vol. 19, no. 4, pp. 853-862, 2004.

[9] G. Raina, "Local bifurcation analysis of some dual congestion control algorithms," Institute of Electrical and Electronics Engineers. Transactions on Automatic Control, vol. 50, no. 8, pp. 1135-1146, 2005.

[10] C. Zhang, Y. Zu, and B. Zheng, "Stability and bifurcation of a discrete red blood cell survival model," Chaos, Solitons and Fractals, vol. 28, no. 2, pp. 386-394, 2006.

[11] Z. He, X. Lai, and A. Hou, "Stability and Neimark-Sacker bifurcation of numerical discretization of delay differential equations," Chaos, Solitons and Fractals, vol. 41, no. 4, pp. 2010-2017, 2009.

[12] H. Su and X. Ding, "Dynamics of a nonstandard finite-difference scheme for Mackey-Glass system," Journal of Mathematical Analysis and Applications, vol. 344, no. 2, pp. 932-941, 2008.

[13] X. Ding and H. Su, "Dynamics of a discretization physiological control system," Discrete Dynamics in Nature and Society, Article ID 51406, 16 pages, 2007.

[14] J. F. Neville and V. Wulf, "Numerical Hopf bifurcation for a class of delay differential equations," Journal of Computational and Applied Mathematics, vol. 115, no. 1-2, pp. 601-616, 2000.

[15] C. Zhang, M. Liu, and B. Zheng, "Hopf bifurcation in numerical approximation of a class delay differential equations," Applied Mathematics and Computation, vol. 146, no. 2-3, pp. 335-349, 2003.

[16] Y. A. Kuznetsov, Elements of Applied Bifurcation Theory, vol. 112 of Applied Mathematical Sciences, Springer, New York, NY, USA, 1995.

[17] J. Dieudonné, Foundations of Modern Analysis, Pure and Applied Mathematics, Vol. X, Academic Press, New York, NY, USA, 1960.

[18] B. D. Hassard, N. D. Kazarinoff, and Y. H. Wan, Theory and Applications of Hopf Bifurcation, vol. 41 of London Mathematical Society Lecture Note Series, Cambridge University Press, Cambridge, UK, 1981.

[19] V. Wulf, Numerical Analysis of Delay Differential Equations Undergoing a Hopf Bifurcation, University of Liverpool, 1999.

[20] S. Wiggins, Introduction to Applied Nonlinear Dynamical Systems and Chaos, vol. 2 of Texts in Applied Mathematics, Springer, New York, NY, USA, 1990. 


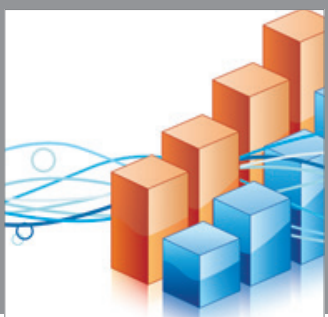

Advances in

Operations Research

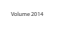

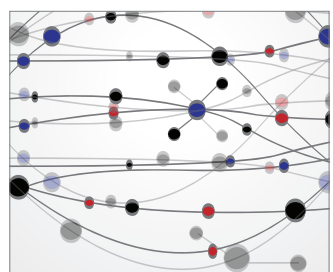

\section{The Scientific} World Journal
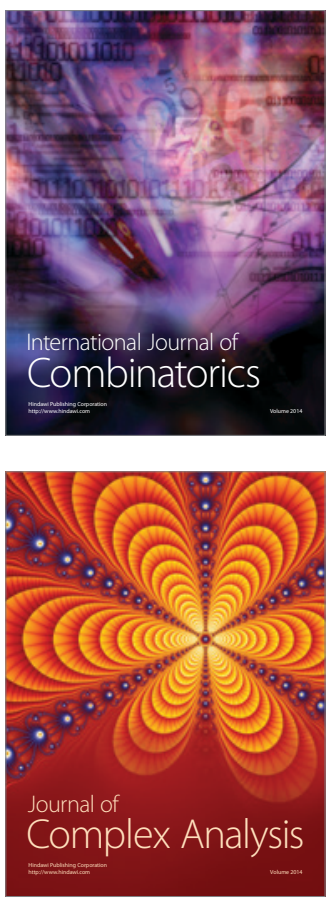

International Journal of

Mathematics and

Mathematical

Sciences
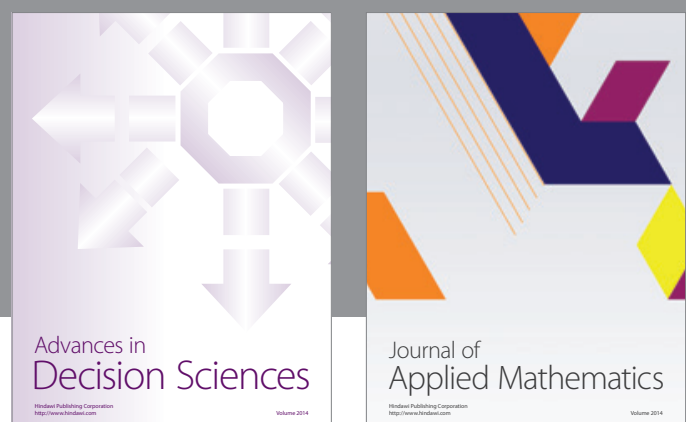

Journal of

Applied Mathematics
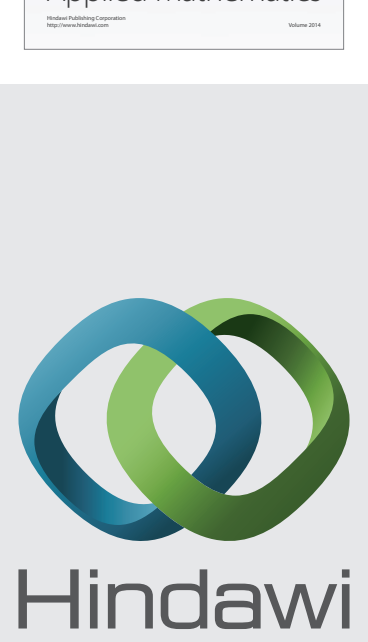

Submit your manuscripts at http://www.hindawi.com
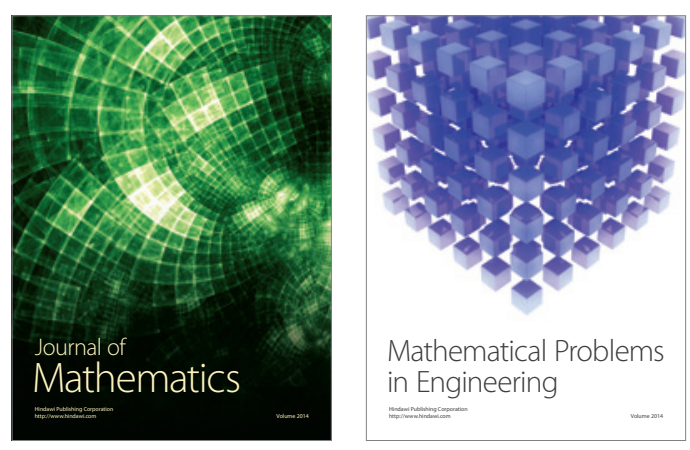

Mathematical Problems in Engineering
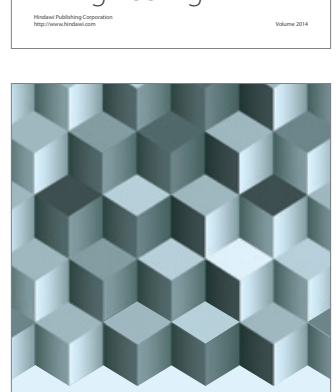

Journal of

Function Spaces
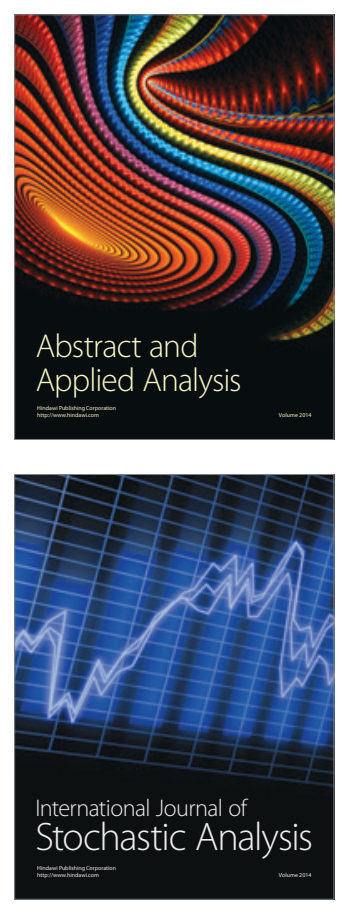

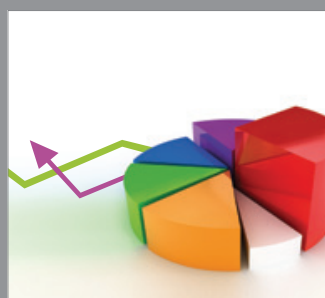

ournal of

Probability and Statistics

Promensencen
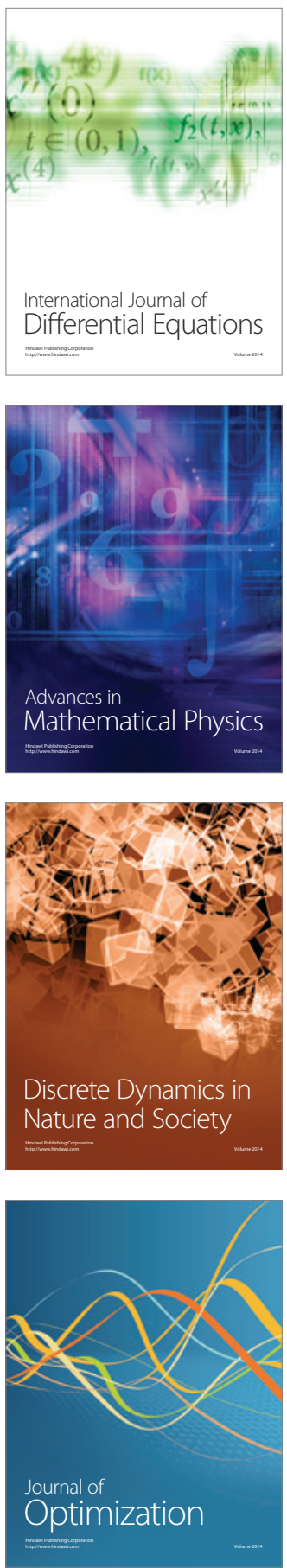